Obere Extremität 2019 $\cdot 14: 229-230$

https://doi.org/10.1007/s11678-019-00548-x

(c) Springer Medizin Verlag $\mathrm{GmbH}$, ein Teil von Springer Nature 2019

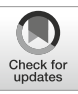

\author{
D. Seybold $\cdot$ K. Wegmann ${ }^{2} \cdot$ A. Ellwein ${ }^{3}$ \\ ' OPND Orthopädische Praxis Klinik Neuss - Düsseldorf, Düsseldorf, Deutschland \\ ${ }^{2}$ Klinik und Poliklinik für Orthopädie und Unfallchirurgie, Universitätsklinikum Köln (AöR), Köln, \\ Deutschland \\ ${ }^{3}$ DIAKOVERE Annastift, Orthopädische Klinik, Medizinische Hochschule Hannover (MHH), Hannover, \\ Deutschland
}

\title{
Posttraumatische Ellenbogenpathologien
}

entsteht [4]. Hierbei wird die Prothese $\mathrm{zu}$ hoch aufgebaut, was unweigerlich $\mathrm{zu}$ einer Druckerhöhung im radialen Gelenk führt und zum kompletten $\mathrm{Ab}$ rieb des Capitulum mit Defektbildung führt. Im Artikel von Burkhart et al. wird das Overlengthening detailliert aufgearbeitet. An klinischen Beispielen wird die Diagnostik erläutert, und der Leser bekommt Techniken angeboten, die Fehlimplantation initial $\mathrm{zu}$ vermeiden.

Ist eine Implantation einer Radiuskopfprothese nicht mehr möglich, da bereits eine relevante Degeneration des $\mathrm{Ca}$ pitulum vorliegt, bietet sich die Anconeusinterposition als Therapieoption an. Die Übersicht von Moro et al. befasst sich eingehend mit dieser Technik, die nicht häufig indiziert ist, aber eine sehr gute Alternative in der komplexen Situation der radiohumeralen Degeneration darstellt.

Die Stabilität des Ellenbogens beruht insbesondere ulnohumeral auf einer hohen knöchernen Konformität; eine Störung der Konformität durch eine insuffiziente Reposition kann rasch zu einer arthrotischen Degeneration des Gelenks führen. Auf der anderen Seite kann es sehr schwer oder gar unmöglich sein, einen signifikant geschädigten Kollateralbandapparat suffizient zu wiederherzustellen. Wenn dann nicht additive Techniken wie Ligamentaugmentierung oder eine externe Stabilisierung eingesetzt werden, kann eine weichteilig begründete Instabilität resultieren. Bei der insgesamt nur geringen Oberfläche der knorpeltragenden Gelenkanteile am Ellenbogen können schon kleinere Defekte zu re-

levanten Konsequenzen führen. Man denke hierbei an persistierende Gelenkstufen nach Osteosynthese der distalen Humerusfraktur, welche zu Subluxationen und drastischen Druckanstiegen auf der Gelenkfläche der Trochlea und des Capitulum führen können. Häufig wird hier im Verlauf eine rasch fortschreitende Degeneration des Gelenks beobachtet. Je nach Konfiguration der vorliegenden Fehlstellung, kann die Stellung und Mechanik des Gelenks durch eine Korrekturosteotomie verbessert werden. Mader et al. stellen anschaulich die Möglichkeiten zur Korrekturosteotomie am distalen Humerus und Unterarm dar. Hierbei handelt es sich um ein anspruchsvolles Gebiet, welches in den letzten Jahren durch die Einführung von dreidimensionalen Planungsprogrammen an Indikationen und an Präzision zugewinnen konnte [5]. Sollte auch mit Korrekturosteotomien eine relevante Verbesserung der knöchernen Situation $\mathrm{zu}$ erreichen sein, findet man im Artikel von Ellwein et al. Lösungsansätze, um die Ellenbogentotalendoprothese bei symptomatischer sekundärer Arthrose nach Frakturversorgung Erfolg versprechend einsetzen zu können.

Die häufigste posttraumatische Pathologie am Ellenbogen ist mit Sicherheit die Gelenksteife [1]. Dies ist darin begründet, dass auch die sekundäre Arthrose oder die Gelenkinstabilität in der gemeinsamen Endstrecke Gelenksteife münden können. Bei der Ellenbogensteife können je nach Ausprägungsgrad viele alltagsrelevante Tätigkeiten nicht mehr ausgeführt werden. Das Führen von Nahrung zum Mund kann genauso unmög- kation ist die Fehlimplantation der Pro-
these, sodass ein sog. „Overlengthening" 
lich werden wie das Bedienen eines Kraftfahrzeugs. Die Fähigkeit zur selbstständigen Versorgung oder die Arbeitsfähigkeit sind dadurch gefährdet, sodass die Steife nicht nur eine häufige, sondern auch eine schwerwiegende posttraumatische Pathologie darstellt. Welche Rolle die Arthroskopie bei der Behandlung der sekundären Ellenbogensteife spielt, wird im Rahmen des vorliegenden Heftes in der englischsprachigen Originalarbeit von Leschinger et al. anhand einer retrospektiven klinischen Studie vermittelt.

Liebe Kolleginnen und Kollegen, wir sind zuversichtlich, Ihnen mit dem vorliegenden Heft praxisrelevante und spannende Inhalte zu bieten, die Sie bei der Behandlung der Patienten mit posttraumatischen Ellenbogenpathologien erfolgreich einbringen können.

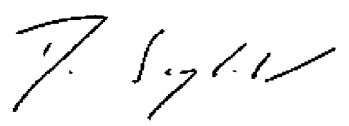

Prof. Dr. Dominik Seybold

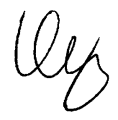

PD Dr. Kilian Wegmann

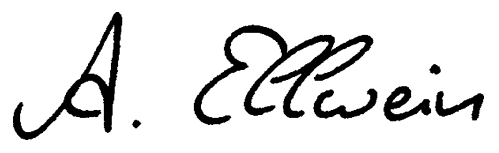

Dr. Alexander Ellwein

\section{Korrespondenzadresse

Prof. Dr. D. Seybold
OPND Orthopädische Praxis
Klinik Neuss - Düsseldorf
Düsseldorf, Deutschland
seybold@
opnd-duesseldorf.de

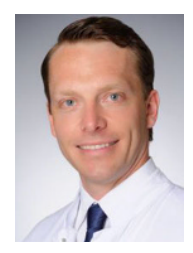

PD Dr. K. Wegmann

Klinik und Poliklinik für Orthopädie und Unfallchirurgie, Universitätsklinikum Köln (AöR) Köln, Deutschland kilian.wegmann@uk-koeln.de

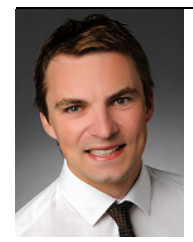

Dr. A. Ellwein

DIAKOVERE Annastift, Orthopädische Klinik, Medizinische Hochschule Hannover (MHH) Hannover, Deutschland alexander.ellwein@ diakovere.de

Interessenkonflikt. D. Seybold, K. Wegmann und A. Ellwein geben an, dass kein Interessenkonflikt besteht.

\section{Literatur}

1. Attum B, Obremskey W (2016) Posttraumatic elbow stiffness. JBJS Rev 4:1-7

2. Laumonerie P, Tibbo ME, Kerezoudis P et al (2018) Short to midterm outcomes of one hundred and seventy one MoPyC radial head prostheses: metaanalysis. Int Orthop 42:2403-2411

3. Mulder PGH, Veltman ES (2016) Radial head arthroplasty - a systematic review. JBJS Rev 4:1-12

4. Frank SG, Grewal R, Johnson J et al (2009) Determination of correct implant size in radial head arthroplasty to avoid overlengthening. JBone Joint Surg Am 91:1738-1746

5. Mauler F, Langguth C, Schweizer A et al (2017) Prediction of normal bone anatomy for the planning of corrective osteotomies of malunited forearm bones using a three-dimensional statistical shape model. J Orthop Res 35:2630-2636 